\title{
SINGLE- VERSUS MULTI-JOINT ISOMETRIC PROTOCOLS TO INDUCE A POST-ACTIVATION POTENTIATION EFFECT ON SQUAT JUMP PERFORMANCE
}

original paper

(1) University School of Physical Education in Wroclaw

DOI: https://doi.org/10.5114/hm.2020.91348

\section{THYAGO GARCIA ${ }^{1}$, JULIANO DAL PUPO ${ }^{1}$, DÉBORA APARECIDA KNIHS ${ }^{1}$, OSVALDO ANDRÉ FURLANETO RODRIGUES ${ }^{1}$, HAIKO BRUNO ZIMMERMANN ${ }^{1}$, JOHNNY PADULO ${ }^{2}$}

${ }^{1}$ Biomechanics Laboratory, Federal University of Santa Catarina, Florianópolis, Brazil

${ }^{2}$ Sport Performance Laboratory, University of Split, Split, Croatia

\begin{abstract}
Purpose. The objective of the study was to analyse the effects of post-activation potentiation induced by single- and multijoint maximal voluntary isometric contractions (MVIC) on squat jump performance.

Methods. Overall, 13 resistance-training males volunteered to participate in the study. Isometric knee extension exercise was chosen to represent single-joint MVIC (MVIC_SJ) and isometric squat to represent multi-joint MVIC (MVIC_MJ). Four experimental protocols were implemented in a randomized order on different days as follows: P1: squat jump performed before and 3 minutes after MVIC_MJ; P2: squat jump performed before and 7 minutes after MVIC_MJ; P3: squat jump performed before and 3 minutes after MVIC_SJ; P4: squat jump performed before and 7 minutes after MVIC_SJ. Jump height, mean power output, peak power output, net impulse, and electromyographic activity of lower limbs were analysed in squat jump before and after the interventions.
\end{abstract}

Results. ANOVA showed that peak power output increased in P1 ( $p=0.04)$. No difference was found for electromyographic activity in any of the protocols.

Conclusions. We concluded that MVIC_MJ performed 3 minutes before the main task (P1) was more effective than MVIC_SJ in optimizing squat jump performance and that the changes in performance were unrelated to muscle activation.

Key words: strength exercises, warm-up, power training, electromyography, biomechanics

\section{Introduction}

The capacity to maximize muscular power is an important outcome in a number of athletic events, such as running and jumping [1]. In the recent decades, several studies have demonstrated that muscle postactivation potentiation (PAP) is a phenomenon that can acutely increase muscular power and, consequently, performance [2-4]. PAP is defined as an optimized muscle state that can enhance force production on subsequent contractions [5], and the activity responsible for inducing PAP is referred to as conditioning contraction (CC) [4]. Utilizing CC is possible to manipulate contractile history and take advantage of both myogenic and neurogenic mechanisms associated with PAP [6]; this may benefit subsequent explosive performance [7].

CC can induce fatigue, which negatively impacts on performance, but can also induce PAP [8, 9]. The efficacy with which CC can stimulate PAP mechanisms and enhance muscular performance depends on the balance between fatigue and potentiation [1]. This balance is strongly influenced by the rest interval between the conditioning and main exercise [10]. The optimal rest time between these two elements remains unclear in the literature, but it seems to be dependent on the level of strength in individuals [11]. According to a meta-analysis [1], short (3-7 minutes) and moderate

Correspondence address: Haiko Bruno Zimmermann, Sports Center of the Federal University of Santa Catarina, Campus Reitor João David Ferreira Lima, s/n - Trindade, Florianópolis - SC, Brazil, Zip code: 88040-900, e-mail: hbzim@hotmail.com protocols to induce a post-activation potentiation effect on squat jump performance. Hum Mov. 2020;21(3):71-80; doi: https:// doi.org/10.5114/hm.2020.91348. 
T. Garcia et al., Post-activation potentiation in squat jump performance

(7-10 minutes) rest period lengths are adequate to optimally augment power output after CC in individuals with resistance training experience. For example, using a 5-repetition-maximum back squat as CC resulted in a significant improvement in standing long jump for up to 10 minutes in female collegiate volleyball players [2]. In addition, a 3-second functional isometric squat performed at $150 \%$ of the 1-repetition-maximum resulted in a $5.1 \%$ increase in jump performance after 4 minutes and a $5.5 \%$ increase after 5 minutes only in resistance-trained subjects [12], corroborating the meta-analysis.

The exercise selection is of primary significance for the efficacy with which CC can stimulate PAP [11]. Regarding the type of muscle contraction, exercises involving the stretch-shortening cycle have been considered effective to stimulate PAP [11, 13]; however, isometric contractions appear to have greater efficiency (i.e., lower metabolic cost, less fatigue, and greater excitability of the neural pool) compared with dynamic contractions $[7,14,15]$. The effect of isometric contraction as CC on vertical jump performance has already been verified in the literature $[7,14,15]$ and it seems to be a good way to induce improvements in performance. However, little attention has been given to the type of exercise used in these activities conditioning maximal voluntary isometric contractions (MVIC).

Considering the number of joints involved, the movement can be classified as single- or multi-joint. Generally, multi-joint exercises are associated with a more complex activation than single-joint ones, with controlled co-contractions of synergistic and antagonistic muscles [16]. Such characteristics may result in distinct activation patterns of the lower limb [17]; therefore, we speculate that single-joint MVIC (MVIC_SJ) and multi-joint MVIC (MVIC_MJ) may have a different impact on the proposed mechanisms of PAP, like reflex potentiation [18] and phosphorylarion of myosin light chains [19]. In addition, it has been postulated that performing multi-joint exercises is advantageous owing to their closer relationship with functional movements and sports events [16].

Several studies have found positive effects on vertical jump performance when multi-joint exercises are used as conditioning activities [12, 14, 20-24]; however, to the best of our knowledge, only one study tested if single- or multi-joint isometric exercises translated into different results on voluntary performance [25]. In this study, the authors showed no enhancement of sprint performance 4 minutes after the conditioning activities composed of 3 repetitions of 3-second isometric knee extension and 3 repetitions of 3 -second isometric squat. In this context, it would be interesting to test if manipulating the exercise (single- vs. multijoint) during MVIC as a CC could lead to different results when the main performance is vertical jump.

Thus, the purpose of this study was to analyse PAP effects induced by MVIC_SJ and MVIC_MJ exercises on squat jump performance. In addition, we aimed to test different recovery times ( 3 or 7 minutes) between $\mathrm{CC}$ and jump performance. The main hypothesis was that there would be a significant increase in vertical jump performance with MVIC_MJ because more muscles would be activated and myogenic and neural mechanisms would be optimized.

\section{Material and methods}

\section{Participants}

A total of 13 resistance-trained males volunteered to participate in this study (age: $24.0 \pm 3.14$ years; height: $1.77 \pm 0.06 \mathrm{~m}$; body mass: $80.72 \pm 3.14 \mathrm{~kg}$ ). The following inclusion criteria were adopted: no history of musculoskeletal injuries in the previous year; at least 2 years of experience in resistance training. The participants were asked to avoid caffeinated drinks for 24 hours before testing and not to perform training sessions involving the lower limbs for 48 hours prior to the evaluations. All received a detailed explanation of the purpose and methods of the study.

\section{Experimental design}

In the present experimental study, we tested the influence of MVIC_SJ and MVIC_MJ (independent variables) on squat jump performance (jump height, power, impulse) and electromyographic (EMG) activity (dependent variables). For this, active individuals with resistance training experience implemented 4 experimental protocols: P1: squat jump performed before and 3 minutes after MVIC_MJ; P2: squat jump performed before and 7 minutes after MVIC_MJ; P3: squat jump performed before and 3 minutes after MVIC_SJ; P4: squat jump performed before and 7 minutes after MVIC_SJ. The time points were selected on the basis of a meta-analysis [1] showing that short (3-7 minutes) and moderate (7-10 minutes) rest period lengths were adequate to optimally augment power output after $\mathrm{CC}$ in individuals with resistance training experience.

The participants visited the laboratory on 4 days to perform the experiment. On the first day, familiarisation with the squat jump protocol and the conditioning 
PAP exercises took place. The familiarisation consisted of verbal explanations of the procedures and performing submaximal exercises focusing on the learning of the squat jump, isometric back squat, and knee extensor chair exercise protocols ( 2 or 3 attempts per exercise were performed, aiming to avoid fatigue or potentiation). Then, the order of protocols $(\mathrm{P} 1 \times \mathrm{P} 2 \times$ P3 $\times$ P4) was randomly assigned with the use of the Latin square methodology. Each protocol was preceded by a warm-up, consisting of 5 minutes of low-intensity cycling exercise (40 RPM; $50 \mathrm{~W}$ ) and 3 sets of 10 hops with a 30-second interval between the sets. The participants then performed one of the selected protocols, involving squat jumps in the precondition, followed by the conditioning exercise and post-potentiation squat jumps. An interval of 48 hours was determined to start a new protocol (Figure 1). We chose to test only one time point per day (3 or 7 minutes) so that the performance test (squat jump) would not induce PAP in the subsequent time point, which could distort the results, leading to a misinterpretation.

\section{Conditioning exercises to induce PAP}

The exercises consisted of the execution of MVIC MJ and MVIC_SJ. For MVIC_MJ, an isometric back squat exercise was performed with a barbell over the subject's shoulders attached to a chain on a wooden platform on the ground (Figure 2a). The subjects performed the exercise in an isometric squat position, with the trunk as upright as possible, with the knees at a $90^{\circ}$ angle, determined by a goniometer [26]. The MVIC_SJ exercise was performed in a knee extensor chair (Progress ${ }^{\circledR}$ Max, TRG Fitness, Brazil), with the arm equipment locked in a position where the subject's knees remained at an angle of approximately $70^{\circ}$ (Figure 2b). This angle was chosen because the peak torque is maximum in this position [27, 28]. Three maximal isometric contractions were performed for each exercise, with a 5-second duration and a 1-minute interval between the sets, as suggested by French et al. [7]. After the isometric exercise, the vertical jumps were performed 3 or 7 minutes later, depending on the protocol.

\section{Vertical jump assessment}

The squat jump was the vertical jump type used in the present study owing to the better control of knee starting angle, which is considered a determinant aspect of the jump performance [26]. The participants performed the squat jump starting from a static position, with the knees at an angle of $90^{\circ}$, the trunk as vertical as possible, and the hands on the waist. The jump was performed without any countermovement, and there was only the positive phase of the agonist muscles involved in the movement. The knee angle of $90^{\circ}$ was determined by using a goniometer. Afterward, a rigid bar was positioned under the participant's hips (posterior thigh) and attached to a vertical support graduated every $1 \mathrm{~cm}$, allowing the adjustment of the bar height in accordance with the specific knee-flexion
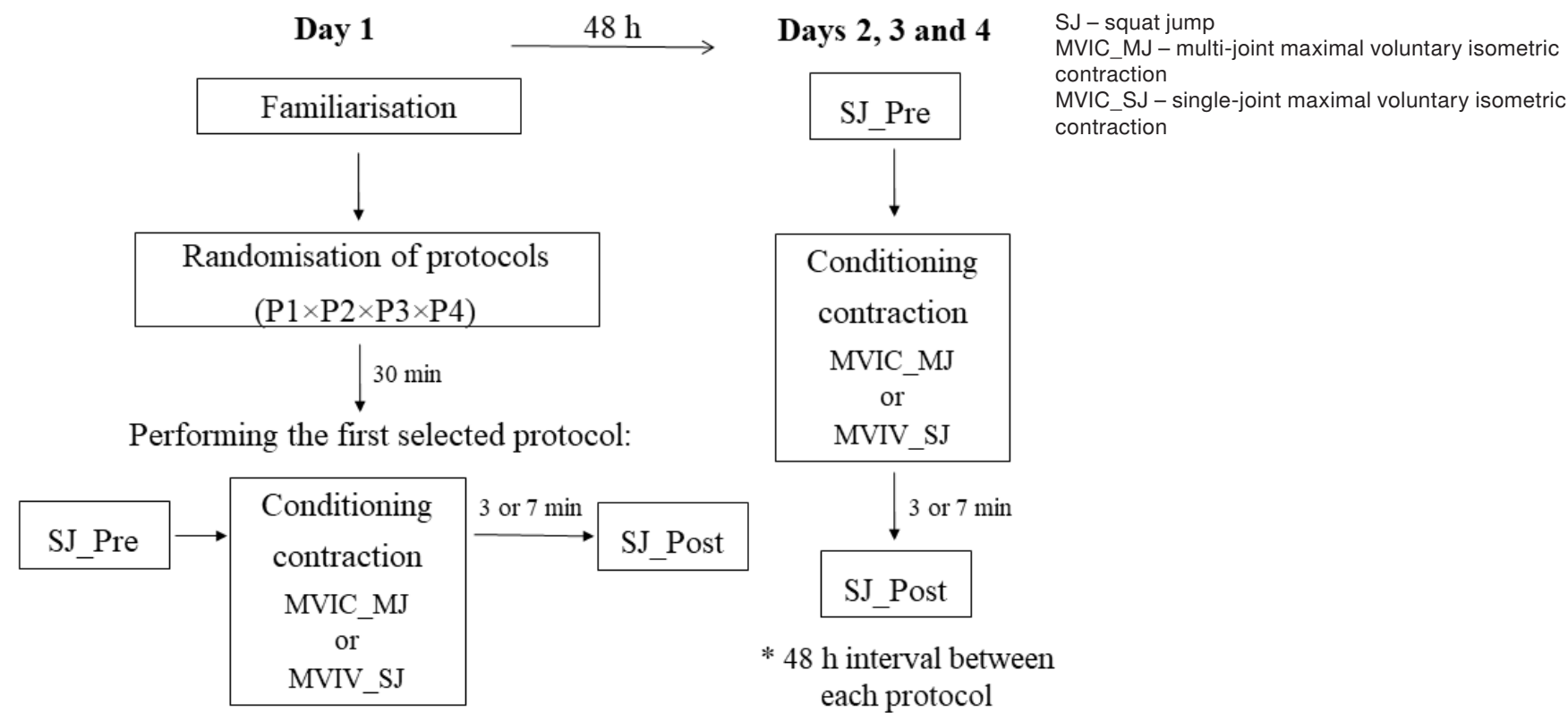

Figure 1. Overview of the experimental protocol 
T. Garcia et al., Post-activation potentiation in squat jump performance

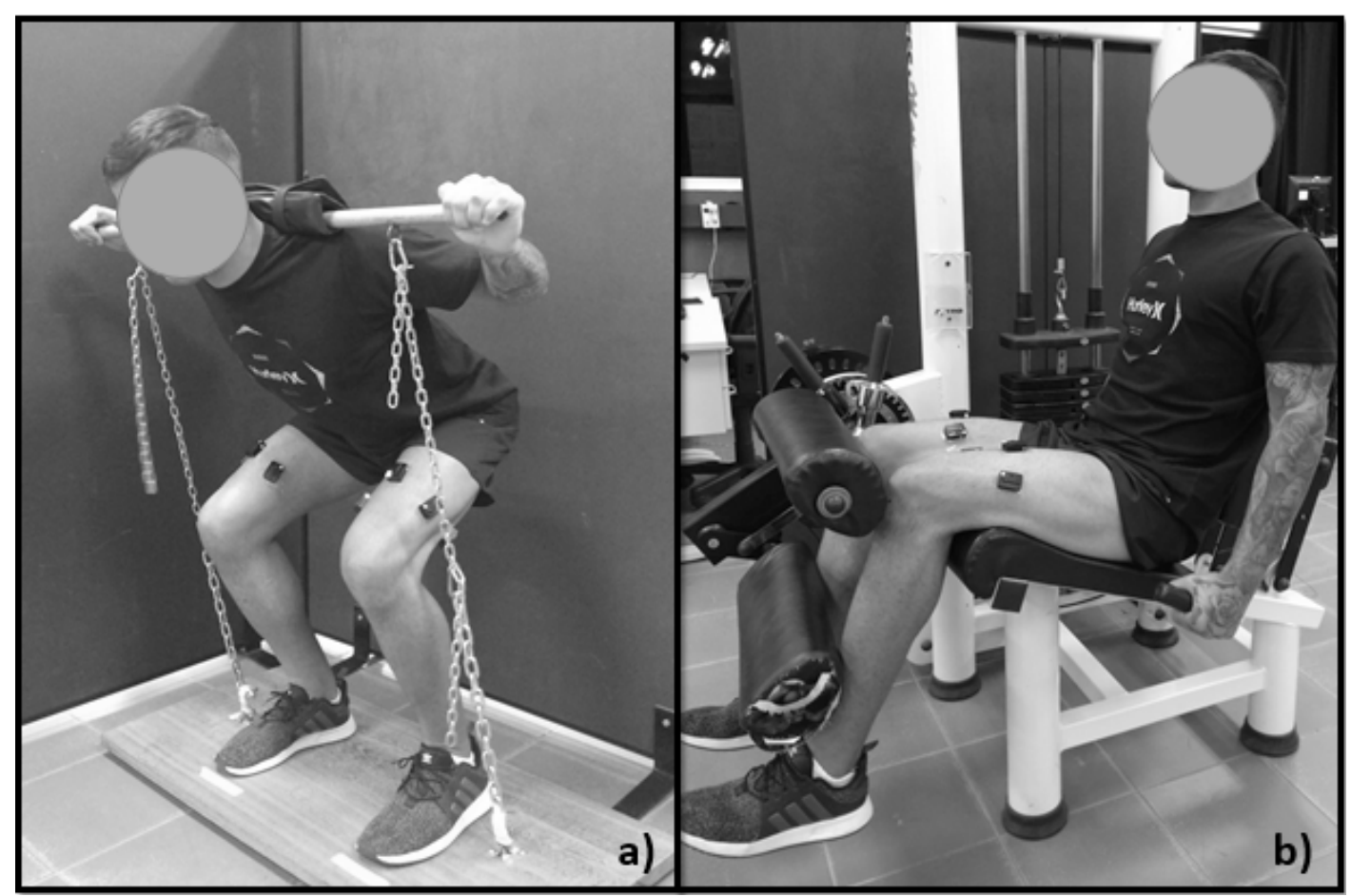

Figure 2. Conditioning exercises: a) isometric back squat; b) isometric knee extension

angle [26]. During the jumps, the subjects squatted until they could feel the bar on their posterior thigh, and then remained static some seconds before performing the positive phase as fast as possible.

The squat jumps were performed on a force platform (Quattro Jump, 9290 AD, Kistler, Winterthur, Switzerland) sampling at $500 \mathrm{~Hz}$. Ground reaction force (GRF) values measured by the platform were acquired by using the Quattro Jump ${ }^{\circledR}$ software. The following variables were calculated: (a) jump height: obtained with the method of double force integration of GRF; (b) power output: calculated by multiplying GRF by velocity (obtained by integration of GRF), and the peak power output and mean power output were considered for analysis; (c) relative net vertical impulse: obtained from the multiplication of GRF over time.

Overall, 5 trials were performed in the pre-condition, prior to each protocol. The coefficient of variation was calculated and 2 attempts with greater variability were disregarded. The average of the remaining 3 trials was considered to be representative of the baseline condition. In the post-condition, only 3 attempts were conducted in order to minimize the effects of recovery.

\section{EMG assessment}

Surface EMG activity of the vastus lateralis, vastus medialis, rectus femoris, and biceps femoris muscles was evaluated during the execution of pre- and post-in- tervention squat jumps. The data collection followed the recommendations of the International Society of Electrophysiology and Kinesiology [29]. Bipolar surface electrodes were positioned parallel to the muscle fibres and fixed with double-sided adhesive tape. Trichotomy and abrasion with 70\% alcohol were performed on the skin before placing the electrodes. The electrodes were not removed.

The EMG signal was collected by using an 8-channel wireless electromyograph (Trigno Wireless EMG System, Delsys, USA) at $2000 \mathrm{~Hz}$. Raw signals were filtered with a combination of $5^{\text {th }}$ order band-pass Butterworth filters between 20 and $500 \mathrm{~Hz}$. The root mean square values of each muscle were calculated during the propulsive phase of the jump. The beginning of the squat jump was considered the moment when GRF was equal to body weight plus 5\%, indicating the point at which the jumper began to accelerate up. Pre- and post-intervention root mean square values were compared, with electrodes remaining undisturbed throughout each protocol.

\section{Statistical analysis}

Data are presented as mean \pm standard deviation. The Shapiro-Wilk test was used to check data distribution. The reliability of vertical jump variables among the 4 pre-test conditions was evaluated by using the following tests: ANOVA for repeated measures, intra- 
class correlation coefficient, and standard error of measurement. ANOVA for repeated measures served to compare the vertical jump variables and EMG between the pre- and post-potentiation conditions for all protocols (P1, P2, P3, P4). Bonferroni's test was applied for post-hoc analysis (pairwise comparison). Statistical significance of the results was set for $p<0.05$ and the statistical power was calculated with the $G^{*}$ Power software. The effect size was calculated confronting pre- vs. post-experimental conditions and classified as small $(\leq 0.40)$, moderate $(0.41-0.80)$, and large (> $0.80)$ [30].

\section{Ethical approval}

The research related to human use has complied with all the relevant national regulations and institutional policies, has followed the tenets of the Declaration of Helsinki, and has been approved by the Ethics Committee of the Federal University of Santa Catarina.

\section{Informed consent}

Informed consent has been obtained from all individuals included in this study.

\section{Results}

Table 1 shows the inter-day reliability of the baseline jump parameters measured before the 4 experimental protocols. There was no significant difference among the measures of pre-protocols 1,2, 3, and 4 for any variable analysed. High intra-class correlation coefficient values were obtained, indicating consistency among measurements; moreover, the standard error of measurement was less than 5\% in all variables. Considering the high reliability found, the average of the 4 pre-tests was used to represent the pre-condition.

Table 2 presents the jump variables assessed before and after the implementation of the PAP protocols. ANOVA showed the effect for peak power output $(\mathrm{F}=$ 2.7; $p=0.04$; power $=0.84$ ), and the post-hoc revealed higher peak power output values after PAP for P1 ( $p=$ 0.01) compared with baseline.

Table 3 shows the effect size and mean differences (absolute and percentage values) for the PAP protocols. In P1, there was a small effect for jump height, mean power output, and peak power output, and moderate effect for net vertical impulse. The percentage changes of the variables for P1 ranged from $2.7 \%$ to $4.4 \%$. In relation to the other protocols (P2, P3, and P4), only small effects were observed, with percentage changes below $1 \%$, except for net vertical impulse for P2 (1.5\%) and P4 (1.88\%).

Figure 3 depicts the EMG activity (root mean square values) of the vastus lateralis, vastus medialis, rectus femoris, and biceps femoris during the squat jump for the 4 experimental protocols. No significant difference was found in any of the protocols when comparing pre- vs. post-PAP in any of the muscles analysed.

Table 1. Inter-day reliability of baseline jump parameters before the PAP protocols

\begin{tabular}{lcccrrrrr}
\hline Parameter & \multicolumn{1}{c}{ Pre_P1 } & Pre_P2 & Pre_P3 & Pre_P4 & Mean $\pm S D$ & F $(p)$ & ICC & SEM $(\%)$ \\
\hline JH $(\mathrm{cm})$ & $47.90 \pm 5.88$ & $47.17 \pm 5.53$ & $47.53 \pm 5.23$ & $47.02 \pm 5.71$ & $47.4 \pm 5.26$ & $0.42(0.74)$ & 0.99 & 3.5 \\
MPO $\left(\mathrm{W} \cdot \mathrm{kg}^{-1}\right)$ & $21.22 \pm 2.85$ & $21.35 \pm 2.48$ & $21.29 \pm 2.65$ & $21.29 \pm 2.65$ & $21.29 \pm 2.65$ & $0.34(0.57)$ & 0.99 & 5.0 \\
PPO $\left(\mathrm{W} \cdot \mathrm{kg}^{-1}\right)$ & $50.63 \pm 5.94$ & $50.85 \pm 5.31$ & $50.73 \pm 6.75$ & $50.74 \pm 5.81$ & $50.74 \pm 5.81$ & $0.04(0.99)$ & 0.96 & 4.5 \\
NI $(\mathrm{N} \cdot \mathrm{m})$ & $2.62 \pm 0.22$ & $2.59 \pm 0.25$ & $2.61 \pm 0.24$ & $2.57 \pm 0.19$ & $2.60 \pm 0.20$ & $0.43(0.74)$ & 0.90 & 5.1 \\
\hline
\end{tabular}

PAP - post-activation potentiation, P1-P4 - PAP protocols, SD - standard deviation, ICC - intra-class correlation coefficient, SEM - standard error of measurement, JH - jump height, MPO - mean power output, PPO - peak power output, NI - net vertical impulse

Table 2. Vertical jump variables before and after the PAP protocols

\begin{tabular}{lcccrrr}
\hline Parameter & Pre & Post_P1 & Post_P2 & Post_P3 & Post_P4 & F $(p)$ \\
\hline JH $(\mathrm{cm})$ & $47.4 \pm 5.26$ & $49.07 \pm 5.26$ & $47.36 \pm 5.4$ & $47.47 \pm 6.9$ & $47.7 \pm 5.8$ & $2.42(0.07)$ \\
MPO $\left(\mathrm{W} \cdot \mathrm{kg}^{-1}\right)$ & $21.29 \pm 2.65$ & $22.05 \pm 2.9$ & $21.42 \pm 2.5$ & $21.3 \pm 2.8$ & $21.3 \pm 2.4$ & $1.71(0.17)$ \\
PPO $\left(\mathrm{W} \cdot \mathrm{kg}^{-1}\right)$ & $50.74 \pm 5.81$ & $52.15 \pm 6.3 *$ & $50.5 \pm 5.3$ & $50.8 \pm 6.2$ & $50.5 \pm 5.0$ & $2.7(0.04)$ \\
NI $(\mathrm{N} \cdot \mathrm{m})$ & $2.60 \pm 0.20$ & $2.72 \pm 0.23$ & $2.64 \pm 0.27$ & $2.58 \pm 0.17$ & $2.65 \pm 0.3$ & $1.83(0.11)$ \\
\hline
\end{tabular}

PAP - post-activation potentiation, P1-P4 - PAP protocols, JH - jump height, MPO - mean power output,

PPO - peak power output, NI - net vertical impulse

* difference from the pre-condition revealed by post-hoc analysis 
T. Garcia et al., Post-activation potentiation in squat jump performance

Table 3. Effect size and mean differences (absolute and \%) for the PAP protocols compared with pre-condition

\begin{tabular}{lccccccccc}
\hline & \multicolumn{2}{c}{ Pre $\times$ P1 } & \multicolumn{2}{c}{ Pre $\times$ P2 } & \multicolumn{2}{c}{ Pre $\times$ P3 } & \multicolumn{2}{c}{ Pre $\times$ P4 } \\
\cline { 2 - 9 } Parameter & ES (CI) & $\Delta$ & ES (CI) & $\Delta$ & ES (CI) & $\Delta$ & ES (CI) & $\Delta$ \\
\hline \multirow{2}{*}{ JH (cm) } & 0.31 & 1.67 & 0.01 & -0.04 & 0.01 & 0.07 & 0.05 & 0.3 \\
& $(-0.47-1.08)$ & $(3.4 \%)$ & $(-0.76-0.78)$ & $(0.08 \%)$ & $(-0.76-0.78)$ & $(0.14 \%)$ & $(-0.72-0.82)$ & $(0.62 \%)$ \\
MPO (W $\left.\cdot \mathrm{kg}^{-1}\right)$ & 0.27 & 0.76 & 0.05 & 0.13 & 0.04 & 0.01 & 0.04 & 0.01 \\
& $(-0.51-1.04)$ & $(3.4 \%)$ & $(-0.72-0.82)$ & $(0.6 \%)$ & $(-0.77-0.77)$ & $(0.04 \%)$ & $(-0.77-0.77)$ & $(0.04 \%)$ \\
PPO (W $\left.\cdot \mathrm{kg}^{-1}\right)$ & 0.22 & 1.41 & 0.04 & -0.24 & 0.01 & 0.06 & 0.04 & -0.24 \\
& $(-0.55-1.00)$ & $(2.7 \%)$ & $(-0.73-0.81)$ & $(0.47 \%)$ & $(-0.76-0.78)$ & $(0.11 \%)$ & $(-0.73-0.81)$ & $(0.47 \%)$ \\
NI (N $\cdot \mathrm{m})$ & 0.56 & 0.12 & 0.17 & 0.04 & 0.11 & -0.02 & 0.20 & 0.05 \\
& $(-0.24-1.32)$ & $(4.4 \%)$ & $(-0.61-0.93)$ & $(1.5 \%)$ & $(-0.66-0.87)$ & $(0.77 \%)$ & $(-0.58-0.96)$ & $(1.88 \%)$ \\
\hline
\end{tabular}

PAP - post-activation potentiation, P1-P4 - PAP protocols, ES - effect size, CI - confidence intervals, JH - jump height, MPO - mean power output, PPO - peak power output, NI - net vertical impulse
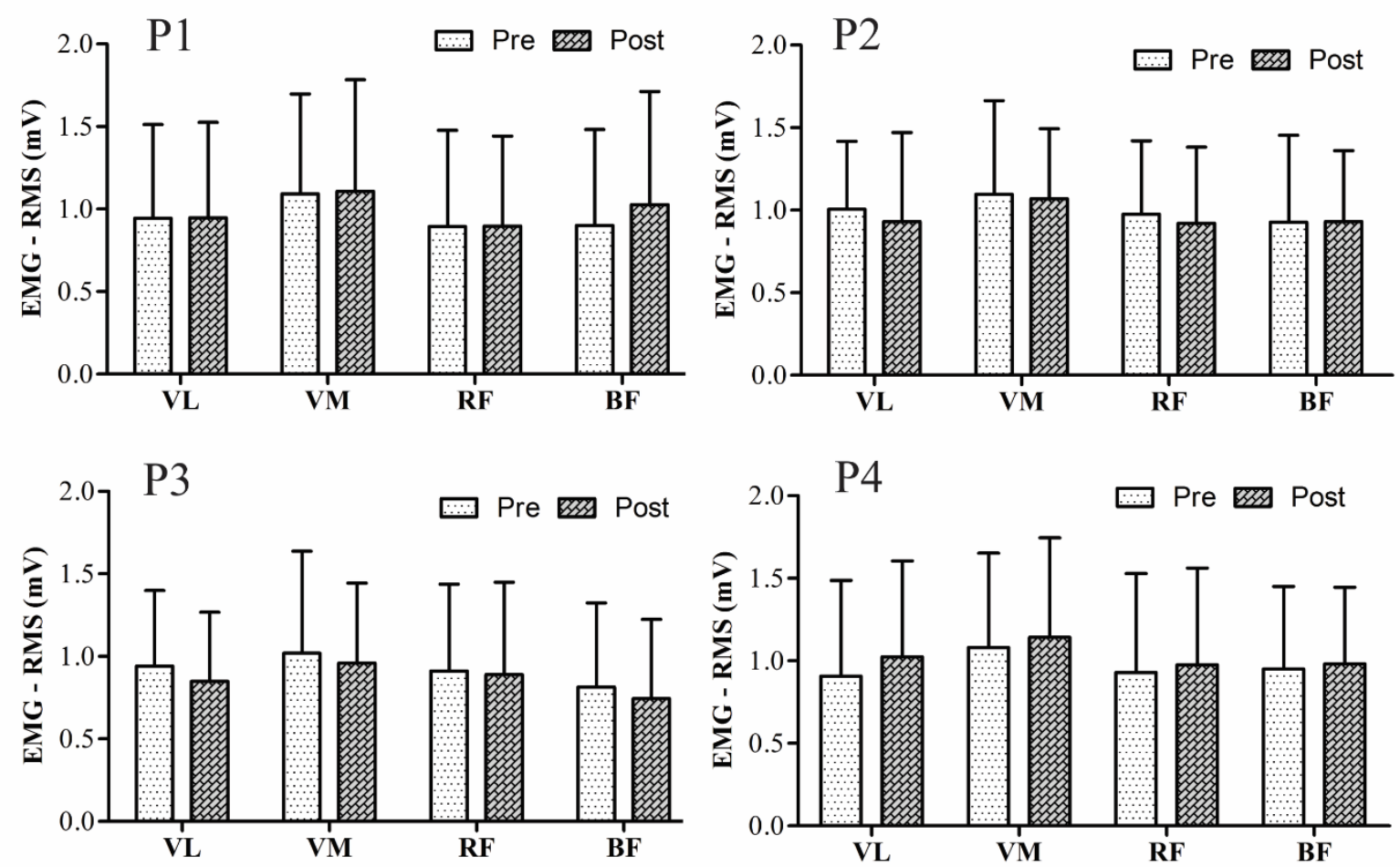

EMG - electromyography, RMS - root mean square, $\mathrm{P} 1$ - post-activation potentiation (PAP) using multi-joint conditioning exercise at 3 minutes, P2 - PAP using multi-joint conditioning exercise at 7 minutes, P3 - PAP using single-joint conditioning exercise at 3 minutes,

P4 - PAP using single-joint conditioning exercise at 7 minutes, VL - vastus lateralis, VM - vastus medialis, RF - rectus femoris, BF - biceps femoris

Figure 3. EMG (RMS) activity of the investigated muscles during squat jump before and after the PAP protocols

\section{Discussion}

The main goal of the present study was to analyse two MVIC interventions (3 repetitions of 5 seconds) performed in different configurations (single- and multi-joint) in the manifestation of positive effects associated with PAP on vertical jump performance. Inferential statistics showed significant effects of P1 (i.e., applying multi-joint MVIC as CC 3 minutes before the squat jump) on peak power output ( $p=0.04$ ).
Therefore, the results of the present study suggest that multi-joint conditioning exercise is more effective than single-joint one to induce PAP in vertical jump through MVIC, thus corroborating the main hypothesis of the study.

One of the main reasons that may explain these findings is that multi-joint conditioning exercises have greater specificity with the vertical jump task. Considering that both CC (PAP inductor exercise) and the squat jump task (performance measure) are multi-joint, they 
will have a similar muscle recruitment pattern and thus a greater transfer of potentiation may be expected [31]. Additionally, while the multi-joint exercise recruits quadriceps, hamstrings, and gastrocnemius muscles, the single-joint exercise focuses only on the quadriceps muscle group; therefore, the effect of PAP tends to be higher when more muscles are involved, possibly owing to the greater number of phosphorylated fibres during multi-joint exercise. On the basis of the principle of specificity, Fletcher [32] recommends that exercises used in the PAP conditioning strategy should mimic the main task as closely as possible. Many studies have already shown that training that applies more specific exercises in terms of motor pattern is more effective to improve performances [33-35].

In the present study, a methodological design was used to test the effect of the type of MVIC as CC on PAP-related improvements. In general, previous studies that used multi-joint interventions as $\mathrm{CC}$ found positive results in vertical jump performance $[2,12,14$, $15,21-23]$. On the other hand, when applying singlejoint exercises in PAP induction, the responses do not seem well established [36]. Consequently, the literature provides evidence showing that multi-joint exercises seem to be more appropriate in PAP induction. We highlight that this was the first study aiming to investigate jump performance after 2 different MVIC as CC, matched in volume, but manipulating the joints involved (multi-joint vs. single-joint) at different recovery time points.

Most studies verified positive effects on vertical jump variables after using squat conditioning exercises. Bogdanis et al. [14] and Rixon et al. [15], for example, found a significant increase in vertical jump height after subjects performed 3 sets of maximum isometric squatting as CC. A similar study was conducted by Seitz et al. [23], but the authors showed that the increase in jump performance (peak power and jump height) after PAP was dependent on the individual strength level. In addition, Esformes and Bampouras [21] stated that squat exercise induced increases in jump height, peak power, and impulse in the vertical jump, but the squat jump exercise was more effective to induce potentiation when performed from a deeper squat position. Comparing different types of jumps as CC (squat jumps and plyometric jumps), Esformes et al. [20] also evidenced that the squat exercise was the most effective to provide vertical jump performance improvements. This is in agreement with our findings with regard to comparison between squatting and knee extension as possible $\mathrm{CC}$, indicating that the best way to induce improvements is with a multi-joint (squat) exercise.

Exercises involving the stretch-shortening cycle have been considered effective strategies to stimulate PAP [11]. However, isometric contractions with short duration (3-5 seconds) are associated with lower metabolic cost and less fatigue compared with dynamic contractions of the same duration, as well as induce greater excitability of the neural pool $[7,14,15]$. Therefore, despite the lower specificity with most of the sports movements, conditioning exercises involving isometric contractions may be considered good strategies for PAP induction [22, 24, 32, 37]. The negative results obtained in previous research using isometric contractions may be possibly explained by an inaccurate balance between PAP and fatigue over the recovery interval [25].

The optimal rest time between the CC and the main performance measure has been extensively studied, but it still remains unclear in the literature. The findings of the present study indicate that the time of 3 minutes is more effective than 7 minutes. Some authors suggest that this interval is subject-dependent $[1,14,24]$; however, a minimum interval of 3 minutes for highly trained athletes and 7 minutes for moderately trained subjects has been suggested for PAP to compensate the fatigue effects [1]. As for the relation between experience time in strength training and the influence of this on PAP, overall, studies have shown that PAP has greater effects in individuals involved in strength training $[1,11,12,14,15,23,38]$. As a result, subjects with an advanced level of strength tend to demonstrate the effects of potentiation earlier (3-9 minutes) than untrained subjects (6-9 minutes) [11, 14]. Comparing trained and untrained men, Berning et al. [12] found improvements in jump performance only in the trained subjects, demonstrating that the potential for using PAP protocols was greater for individuals with training experience.

Our results corroborate previous studies with regard to PAP-fatigue relationship. French et al. [7] observed that there was an increase of drop jump (5\%) and isokinetic knee extension (6.1\%) immediately after 3 sets of 3-second MVIC; however, they also noted jump performance decrement immediately after 3 sets of 5-second MVIC, possibly owing to the greater fatigue generated in this CC, suppressing the effects of PAP. In the present study, using 3 sets of 5-second MVIC, we found improvements of peak power output at the 3-minute recovery time point, which is in agreement with our initial expectations. Likewise, Lim and Kong 
T. Garcia et al., Post-activation potentiation in squat jump performance

[25], applying 3 sets of 3-second MVIC, did not report improvements in the performance of short sprints after any protocol 4 minutes after the CC. It can be thus speculated that the CC used by the mentioned authors did not generate enough PAP, possibly because of a lower volume or too long recovery time, since our results showed improvement in performance after 3 sets of 5-second MVIC at the 3-minute recovery time point. Collectively, these results demonstrate the influence of CC volume and optimal recovery time on the expression of positive results associated with PAP effects.

The results of the present study showed that there were no effects of potentiation on muscle activation in any of the muscles evaluated. It was hypothesised that there would be an increase in EMG activity during the squat jump after the $\mathrm{CC}$, since one of the possible mechanisms of muscle potentiation is an increase in recruitment of motor units [39]. Some studies in the literature corroborate our findings. French et al. [7], for example, verified that the vastus medialis muscle activation did not change during knee extension between the pre- and post-intervention through MVIC. Esformes et al. [40], despite analysing different musculature, also did not observe changes in the EMG activity of the pectoralis major and brachial triceps during the ballistic bench press throw after CC by 4 different types of contractions: concentric, isometric, eccentric, and concentric-eccentric. On the other hand, some studies have shown divergent results. Fletcher [32] found an increase in the EMG activity of the biceps femoris, rectus femoris, and gastrocnemius during countermovement, squat, and drop jumps after a protocol consisting of warm-up exercises on cycle ergometer, dynamic stretching, and squat exercises (CC). Hence, the inconclusive results of EMG after CC indicate that the increase in jump performance is not always caused by an increase in recruitment of motor units [40]. It is suggested here that the potentiation may induce efficiency in the vertical jump task, i.e., increase the performance (jump height, power) without the need to raise the level of muscle activation, probably because of intramuscular changes associated with phosphorylation of myosin regulatory light chains and a more effective cross bridge cycling [19]. It has been proposed that this phosphorylation would lead to an increase in the rate of transition from a non-forcegenerating state to a force-generating state of the cycling cross bridges [41] and that would result in an optimized force and power production in the subsequent activity [22].

Thus, on the basis of our results, coaches and athletes are advised to use isometric multi-joint exercises for increasing vertical jumping performance, providing more specificity and greater transfer to the main task. For future investigations, it is recommended to evaluate the effect of specificity of PAP-inducing exercises on different types of power sports modalities, such as horizontal jumps, sprints, and throws.

\section{Conclusions}

In conclusion, the results of the present study indicate that a multi-joint conditioning exercise, using maximal isometric squatting, is more effective than a single-joint isometric knee extension exercise to induce improvement in squat jump performance (i.e., peak power output). No changes were observed in muscular activity, which indicates that the increase in squat jump performance observed for P1 was unrelated to neural activation. In addition, for resistancetrained subjects, adopting a shorter time interval (3 minutes) between the $\mathrm{CC}$ and main activity (squat jump) seems to be more effective than a longer interval (7 minutes).

\section{Acknowledgements}

The authors wish to acknowledge all volunteer participants for their collaboration.

\section{Disclosure statement}

No author has any financial interest or received any financial benefit from this research.

\section{Conflict of interest}

The authors state no conflict of interest.

\section{References}

1. Wilson JM, Duncan NM, Marin PJ, Brown LE, Loenneke JP, Wilson SM, et al. Meta-analysis of postactivation potentiation and power: effects of conditioning activity, volume, gender, rest periods, and training status. J Strength Cond Res. 2013;27(3):854-859; doi: 10.1519/JSC.0b013e31825c2bdb.

2. Ah Sue R, Adams KJ, DeBeliso M. Optimal timing for post-activation potentiation in women collegiate volleyball players. Sports. 2016;4(2):27; doi: 10.3390/ sports 4020027.

3. Docherty D, Hodgson MJ. The application of postactivation potentiation to elite sport. Int J Sports Physiol Perform. 2007;2(4):439-444; doi: 10.1123/ijspp.2.4.439.

4. Tillin NA, Bishop D. Factors modulating post-activation potentiation and its effect on performance of subsequent explosive activities. Sports Med. 2009;39(2): 147-166; doi: 10.2165/00007256-200939020-00004.

5. Hodgson M, Docherty D, Robbins D. Post-activation potentiation: underlying physiology and implications 
for motor performance. Sports Med. 2005;35(7):585595; doi: 10.2165/00007256-200535070-00004.

6. Jordan M, Norris S, Smith D, Herzog W. Acute effects of whole-body vibration on peak isometric torque, muscle twitch torque and voluntary muscle activation of the knee extensors. Scand J Med Sci Sports. 2009;20(3): 535-540; doi: 10.1111/j.1600-0838.2009.00973.x.

7. French DN, Kraemer WJ, Cooke CB. Changes in dynamic exercise performance following a sequence of preconditioning isometric muscle actions. J Strength Cond Res. 2003;17(4):678-685; doi: 10.1519/15334287(2003)017<0678:CIDEPF>2.0.CO;2.

8. Sale DG. Postactivation potentiation: role in human performance. Exerc Sport Sci Rev. 2002;30(3):138143; doi: 10.1097/00003677-200207000-00008.

9. Rassier DE, MacIntosh BR. Coexistence of potentiation and fatigue in skeletal muscle. Braz J Med Biol Res. 2000;33(5):499-508; doi: 10.1590/S0100879X2000000500003.

10. Kilduff LP, Bevan HR, Kingsley MI, Owen NJ, Bennett MA, Bunce PJ, et al. Postactivation potentiation in professional rugby players: optimal recovery. J Strength CondRes. 2007;21(4):1134-1138; doi: 10.1519/R-20996.1.

11. Seitz LB, Haff GG. Factors modulating post-activation potentiation of jump, sprint, throw, and upper-body ballistic performances: a systematic review with meta-analysis. Sports Med. 2016;46(2):231-240; doi: 10.1007/s40279-015-0415-7.

12. Berning JM, Adams KJ, DeBeliso M, Sevene-Adams PG, Harris C, Stamford BA. Effect of functional isometric squats on vertical jump in trained and untrained men. J Strength Cond Res. 2010;24(9):22852289; doi: 10.1519/JSC.0b013e3181e7ff9a.

13. Bogdanis GC, Tsoukos A, Veligekas P. Improvement of long-jump performance during competition using a plyometric exercise. Int J Sports Physiol Perform. 2017;12(2):235-240; doi: 10.1123/ijspp.2016-0116.

14. Bogdanis GC, Tsoukos A, Veligekas P, Tsolakis C, Terzis G. Effects of muscle action type with equal impulse of conditioning activity on postactivation potentiation. J Strength Cond Res. 2014;28(9):2521-2528; doi: 10.1519/JSC.0000000000000444.

15. Rixon KP, Lamont HS, Bemben MG. Influence of type of muscle contraction, gender, and lifting experience on postactivation potentiation performance. J Strength CondRes.2007;21(2):500-505; doi:10.1519/R-18855.1.

16. Mayer F, Schlumberger A, van Cingel R, Henrotin Y, Laube W, Schmidtbleicher D. Training and testing in open versus closed kinetic chain. Isokinet Exerc Sci. 2003;11(4):181-187; doi: 10.3233/IES-2003-0154.

17. Escamilla RF, Fleisig GS, Zheng N, Barrentine SW, Wilk KE, Andrews JR. Biomechanics of the knee during closed kinetic chain and open kinetic chain exercises. Med Sci Sports Exerc. 1998;30(4):556-569; doi: 10.1097/00005768-199804000-00014.

18. Folland JP, Wakamatsu T, Fimland MS. The influence of maximal isometric activity on twitch and H-reflex potentiation, and quadriceps femoris performance. Eur
J Appl Physiol. 2008;104(4):739-748; doi: 10.1007/ s00421-008-0823-6.

19. Vandenboom R. Modulation of skeletal muscle contraction by myosin phosphorylation. Compr Physiol. 2017; 7(1):171-212; doi: 10.1002/cphy.c150044.

20. Esformes JI, Cameron N, Bampouras TM. Postactivation potentiation following different modes of exercise. J Strength Cond Res. 2010;24(7):1911-1916; doi: 10.1519/ JSC.0b013e3181dc47f8.

21. Esformes JI, Bampouras TM. Effect of back squat depth on lower-body postactivation potentiation. J Strength Cond Res. 2013;27(11):2997-3000; doi: 10.1519/ JSC.0b013e31828d4465.

22. Fukutani A, Takei S, Hirata K, Miyamoto N, Kanehisa H, Kawakami Y. Influence of the intensity of squat exercises on the subsequent jump performance. J Strength Cond Res. 2014;28(8):2236-2243; doi: 10.1519/ JSC.0000000000000409.

23. Seitz LB, de Villarreal ES, Haff GG. The temporal profile of postactivation potentiation is related to strength level. J Strength Cond Res. 2014;28(3):706-715; doi: 10.1519/JSC.0b013e3182a73ea3.

24. Wyland TP, Van Dorin JD, Reyes GF. Postactivation potentation effects from accommodating resistance combined with heavy back squats on short sprint performance. J Strength Cond Res. 2015;29(11):3115-3123; doi: 10.1519/JSC.0000000000000991.

25. Lim JJ, Kong PW. Effects of isometric and dynamic postactivation potentiation protocols on maximal sprint performance. J Strength Cond Res. 2013;27(10):27302736; doi: 10.1519/JSC.0b013e3182815995.

26. Gheller RG, Dal Pupo J, Ache-Dias J, Detanico D, Padulo J, dos Santos SG. Effect of different knee starting angles on intersegmental coordination and performance in vertical jumps. Hum Mov Sci. 2015;42:7180; doi: 10.1016/j.humov.2015.04.010.

27. Knapik JJ, Wright JE, Mawdsley RH, Braun J. Isometric, isotonic, and isokinetic torque variations in four muscle groups through a range of joint motion. Phys Ther. 1983;63(6):938-947; doi: 10.1093/ptj/63.6.938.

28. Brughelli M, Cronin J, Nosaka K. Muscle architecture and optimum angle of the knee flexors and extensors: a comparison between cyclists and Australian rules football players. J Strength Cond Res. 2010;24(3):717721; doi: 10.1519/JSC.0b013e318197009a.

29. Merletti R. Standards for reporting EMG data. International Society of Electrophysiology and Kinesiology; 1999.

30. Cohen J. Statistical power analysis for the behavioral sciences. New York: Lawrence Erlbaum Associates; 1988.

31. Reilly T, Morris T, Whyte G. The specificity of training prescription and physiological assessment: a review. J Sports Sci. 2009;27(6):575-589; doi: 10.1080/02640 410902729741.

32. Fletcher IM. An investigation into the effect of a pre-performance strategy on jump performance. J Strength Cond Res. 2013;27(1):107-115; doi: 10.1519/JSC.0b0 13e3182517ffb. 


\section{HUMAN MOVEMENT}

T. Garcia et al., Post-activation potentiation in squat jump performance

33. Crewther BT, Kilduff LP, Cook CJ, Middleton MK, Bunce PJ, Yang GZ. The acute potentiating effects of back squats on athlete performance. J Strength Cond Res. 2011;25(12):3319-3325; doi: 10.1519/JSC.0b013 e318215f560.

34. Kitai TA, Sale DG. Specificity of joint angle in isometric training. Eur J Appl Physiol Occup Physiol. 1989;58(7):744-748; doi: 10.1007/BF00637386.

35. Tønnessen E, Shalfawi SA, Haugen T, Enoksen E. The effect of 40-m repeated sprint training on maximum sprinting speed, repeated sprint speed endurance, vertical jump, and aerobic capacity in young elite male soccer players. J Strength Cond Res. 2011;25(9):23642370; doi: 10.1519/JSC.0b013e3182023a65.

36. Smith JC, Fry AC. Effects of a ten-second maximum voluntary contraction on regulatory myosin light-chain phosphorylation and dynamic performance measures. J Strength Cond Res. 2007;21(1):73-76; doi: 10.1519/ 00124278-200702000-00014.

37. Chatzopoulos DE, Michailidis CJ, Giannakos AK, Alexiou KC, Patikas DA, Antonopoulos CB, et al. Postactivation potentiation effects after heavy resistance exercise on running speed. J Strength Cond Res. 2007;21(4): 1278-1281; doi: 10.1519/R-21276.1.

38. Batista MA, Roschel H, Barroso R, Ugrinowitsch C, Tricoli V. Influence of strength training background on postactivation potentiation response. J Strength Cond Res. 2011;25(9):2496-2502; doi: 10.1519/JSC.0b013e 318200181b.

39. Guellich A, Schmidtbleicher D. MVC-induced shortterm potentiation of explosive force. New Stud Athl. 1996;11(4):67-81.

40. Esformes JI, Keenan M, Moody J, Bampouras TM. Effect of different types of conditioning contraction on upper body postactivation potentiation. J Strength Cond Res.2011;25(1):143-148; doi: 10.1519/JSC.0b013e3181 fef7f3.

41. Baudry S, Klass M, Duchateau J. Postactivation potentiation of short tetanic contractions is differently influenced by stimulation frequency in young and elderly adults. Eur J Appl Physiol. 2008;103(4):449-459; doi: 10.1007/s00421-008-0739-1. 